\title{
Soft Fingertips with Tactile Sensing and Active Deformation for Robust Grasping of Delicate Objects
}

\author{
Liang $\mathrm{He}^{1}$, Qiujie $\mathrm{Lu}^{1}$, Sara-Adela $\mathrm{Abad}^{2,3}$, Nicolas Rojas ${ }^{1}$, and Thrishantha Nanayakkara ${ }^{1}$
}

\begin{abstract}
Soft fingertips have shown significant adaptability for grasping a wide range of object shapes thanks to elasticity. This ability can be enhanced to grasp soft, delicate objects by adding touch sensing. However, in these cases, the complete restraint and robustness of the grasps have proved to be challenging, as the exertion of additional forces on the fragile object can result in damage. This paper presents a novel soft fingertip design for delicate objects based on the concept of embedded air cavities, which allow the dual ability of tactile sensing and active shapechanging. The pressurized air cavities act as soft tactile sensors to control gripper position from internal pressure variation; and active fingertip deformation is achieved by applying positive pressure to these cavities, which then enable a delicate object to be kept securely in position, despite externally applied forces, by form closure. We demonstrate this improved grasping capability by comparing the displacement of grasped delicate objects exposed to high-speed motions. Results show that passive soft fingertips fail to restrain fragile objects at accelerations as low as $0.1 \mathbf{~ m} / \mathbf{s}^{\mathbf{2}}$, in contrast, with the proposed fingertips delicate objects are completely secure even at accelerations of more than $5 \mathrm{~m} / \mathrm{s}^{2}$.
\end{abstract}

Index Terms-Soft Sensors and Actuators, Grasping, Gripper and Other End-Effectors.

\section{INTRODUCTION}

The need for robotic grasping and manipulating soft objects without damage has been growing in farming, industrial, and household environments [1]. Soft and fragile objects such as fruits, vegetables, or other non-rigid food products tend to either break or deform if too much force is applied [2]. Both the agricultural sector and the food industry are currently urging for such grippers in the continuous automation of their processes, for increased efficiency, hygiene, and qualitye.g., [3], Human workers can easily handle delicate products without crushing them for tasks such as gripping, positioning, orienting, and placing. In contrast, robot grippers face great

Manuscript received: September, 10, 2019; Revised November, 17, 2019; Accepted January, 20, 2020.

This paper was recommended for publication by Editor Kyu-Jin Cho upon evaluation of the Associate Editor and Reviewers' comments. *This work was supported in part by the Engineering and Physical Sciences Research Council grant EP/R020833/1.

${ }^{1}$ Liang He, Qiujie Lu, Nicolas Rojas, and Thrishantha Nanayakkara are with the Dyson School of Design Engineering, Imperial College London, 25 Exhibition Road, London SW7 2DB, UK. L. he1 7 a imperial.ac.uk

${ }^{2}$ Sara-Adela Abad is with the Department of Mechanical Engineering, University College London, London, UK s. abad-guaman@ucl . ac . uk

3 Sara-Adela Abad is also with the Institute for Applied Sustainability Research, Av. Granados E13-55 e Isla Marchena, No.44, Quito, 170503, Ecuador.

Digital Object Identifier (DOI): see top of this page.
Fig. 1. Embedded air cavities in the proposed soft fingertips allow the dual ability of soft tactile sensing, via internal pressure variation, and active deformation, via positive pressure. The active shape-changing allows keeping delicate objects securely in position despite external forces being applied.

difficulties to grasp soft and fragile objects with different size, stiffness, and shape [4]. Natural food products like fruits, for instance, tend to get bruised when high contact pressure is applied on the contact point [5]. In addition, the modeling of the contact point and the gripper is limited due to the shape and ripeness variation of natural fruits. The grasping also needs to be robust enough to be manipulated in high speed for production efficiency. The challenge is how to ensure precision robust grasping with controllable applied force on soft objects.

Currently, rigid contact grippers [6], soft robotic grippers [7], and air with suction cups [8] are designed to be used in the food industry for pick and place tasks. Rigid grippers have the advantages of high precision control with the ease of integrating tactile, position, and torque sensors [9]. A stable grasping force and dexterous manipulation of the object can be achieved with force and position feedback [10]. However, rigid grippers are lacking adaptability, and they are prone to cause damages when manipulating very fragile and soft objects. Conversely, soft grippers such as the starfish gripper with the embedded pneumatic networks (PneuNets) [11] and phase changing grippers like the universal gripper with granular jamming material [12] show high resilience and compliance. The high adaptability of soft robotic grippers indeed increased the performance in grasping delicate food products with lower contact pressure, yet the precise modeling and robustness are very challenging compared to rigid grippers [13]. In addition, most grasping tasks with soft grippers are enabled by power grasp, which disabled the capability for in-hand and precision 
manipulation [14]. Specialized grasping technologies based on airflow such as suction cups and Bernoulli principle grippers are possible to handle objects with different sizes and work with multiple objects at the same time [15], [8]. However, the airflow-based technologies work poorly with products that have rough surfaces, porous structure, and irregular shapes. The product surface can also get damaged or marked during grasping and manipulation.

One solution to achieve robust soft object grasping is to enable form closure and tactile sensing with fingertips that can change the morphology. Form closure is created when the object is fully constrained by contacts of the grippers, irrespective of the magnitude of the forces [16], [17]. Thus, the required force to prevent the object from escaping can be significantly reduced in industrial pick and placing tasks compared to two-point pitch grasp [18] which relies on friction (force closure). The increased contact areas between the object and gripper can also improve the grasping stability as shown in [19], where the shape of the fingertips are designed to adapt the object based on contact primitives. Tactile sensors, such as capacitive [20], optical [10], and piezoelectric [21] sensors are commonly being employed on the fingers for sensing to detect grasping force and increase accuracy [22], [10]. Fingertip designs with embedded sensors can also achieve high resolution and relatively soft contact when interacting with the object [23], [24]. However, conventional tactile sensors are difficult to integrate with high softness grippers and soft fingertips that can produce active deformation.

In this paper, we propose a novel fingertip design that has the dual ability of tactile sensing and active shape-changing (Fig. 1) with the same set of pneumatic-controlled hardware. The fingertips are integrated and tested with a traditional grasper with four-bar-linkage fingers based on the GR2 gripper [25]. The grasper itself without the designed fingertips is inappropriate for soft object grasping as a high contact force is needed for securing the object in hand. Our aim is to combine the advantages of soft robot design with a rigid gripper to change the soft fingertip geometry via positive pressures and use pressure variations for tactile sensing. By active changing the shape of the fingertips to adapt to the object shape, implementations such as 2D form-closure grasping can be created in regard to the object sizes. Combining the dual function of the soft fingertips allows the gripper to be able to grasp soft and delicate objects with low contact force, while being robust enough for high-speed manipulations.

The rest of the paper is organized as follows. Section II introduces the design and control of the dual-soft fingertips and modified gripper. Experiment results of crushing tests with sensorised soft cylindrical objects of different size and escaping tests with these at high speed are detailed in section III and IV, respectively. Finally, section V provides a conclusion and discussion of the work.

\section{Fingertip Design And Control}

\section{A. Fingertip design}

The fingertip structure was designed with the consideration of the planar grasping motion for demonstrating the proposed
Fig. 2. (a) Schematics of the dual-function fingertip design. (b) Fingertip thickness $w$ and (c) shore hardness variation of inflation (blue) and deflation (red) tests. The $\mathrm{x}$-axis label of b is the same as c. (d) Sensor output values versus applied surface load for fingertips of different inflated pressure.Three lines per inflated condition were shown with loading and unloading test cycles.

dual-functionality. The soft fingertip is designed with silicone rubber (Ecoflex 0030 with part A, part B mixing ratio of 1:1, from Smooth-on, USA) to ensure safe interaction with soft objects. Two half-cylindrical air cavities made from the same silicone rubber are created and cast inside the soft fingertip for sensing and inflation. The base of the soft fingertip is 3D printed with VeroClear on a Stratasys Object 260 3D printer. The structure of the base is designed to ensure the silicone fingertip fully secures on the base and grow in the desired direction during inflation. Fig. 2a. shows the schematics of the dual-function fingertip design. This figure also demonstrates a scenario where the fingertips are inflated to adapt to the object shape actively, and a 2D form-closure grasping can be therefore achieved. With different inflated air cavity sizes, the fingertips can be controlled to adapt to the grasping object. According to the ideal gas law, $P V=n R T$ (where, $P, V$, and $T$ are the pressure, volume, and absolute temperature, $n, R$ are the number of moles of gas and the ideal gas constant), the internal air pressure shows a co-relation to the volume change of the air cavity when $n, R, T$ are constant. Considering the incompressibility of silicone, the volume change of the air cavity caused by external force can be measured with a pressure sensor. Previous work [26] about pneumatic deformation 
Fig. 3. (a) Kinematic chain of the gripper during grasping task. P1, P2, P3, and P4 are revolute joints on the left gripper. P5 is the middle point of the fingertip rigid surface. P6 is the middle point of the silicone surface of the fingertip. $\theta$ is the input angle to close/open the mechanism. $\rho$ is the fixing angle of the central triangular link after locked in place. D is the distance between two grippers measured from the middle points. The gripper moves symmetric during the grasping task in this study. (b) Sample data of the pressure reading of the four air cavites when grasping a 30mm cylinder. The four subplots are represented from the four sensing air cavities (L1, L2, R1, and R4), respectively.

sensing confirms that the air cavity can be used as a force sensor with the reading from pressure sensors.

We use the embedded air cavities as soft tactile sensors to control the gripper position with the internal pressure data. When the air cavity is inflated, the deformation of the air cavity causes shape change of the gripper's fingertip. The mechanical properties (size and hardness) of the fingertip were tested and shown in Fig. 2. Thickness $w$ and shore hardness were collected at the widest part of the fingertip with a caliper and a type C Durometer (resolution of $0.5 \mathrm{HC}$ ), respectively. For a single trial, the fingertip was inflated by step around $5 \mathrm{kPa}$ to measure those mechanical properties, same as the deflation. The curves represent the average data of 5 trials of inflation/deflation tests in a continuous cycle. The fingertip thickness $w$ increases from $22 \mathrm{~mm}$ to $29 \mathrm{~mm}$ and the shore hardness of the fingertip increases from $20 \mathrm{HC}$ to $33.5 \mathrm{HC}$ during inflation $(102 \mathrm{kPa}$ to $150 \mathrm{kPa})$. The measured shore hardness of the original Ecoflex 0030 is $25 \mathrm{HC}$. The fingertip exhibits a hardness change during inflation from softer to stiffer compared to original fingertips. Fig. 2 also shows that the hysteresis is 0.005 for thickness and -0.062 for shore hardness as a consequence of material hysteresis. The maximum measured variability was $0.9 \mathrm{~mm}$ for thickness and 2.3 HC for shore hardness. The sensitivity of the tactile sensor was evaluated by applying a load to the center of one of the fingertip cavities. A 3D printed probe with the tip diameter of $20 \mathrm{~mm}$ was mounted on an ANT130 XY-stage (Aerotech Inc., accuracy of $2.5 \mu \mathrm{m}$ ) with an ATI Mini40-E Force/Torque sensor (SI-40-2, ATI Industrial Automation, USA, resolution of $0.02 \mathrm{~N}$ ) attached to perform the calibration. The load was applied incrementally until the sensor reading saturated. Due to the maximum intake load of the XY-stage, the maximum force was recorded at around $20 \mathrm{~N}$. A clear increase of sensing range was observed when inflating the air cavity. The maximum sensing range of the non-inflated state cavity is around $10 \mathrm{~N}$,

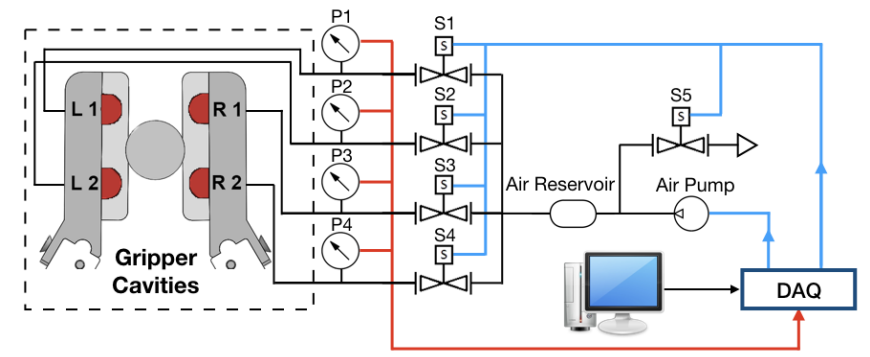

Fig. 4. Pneumatic diagram for the fingertip control. All 4 pressurized air cavities are controlled and measured independently. The dashed square indicates the components of the fingertips while pressure sensors, solenoid valves, the air reservoir, and the air pump are connected externally. Black lines indicate the pneumatic connections, red lines indicate the sensors signals, and blue lines indicate control signals.

while the maximum range increased to more than $20 \mathrm{~N}$ when the air cavity is inflated to $50 \mathrm{kPa}$. The load was also gradually removed to evaluate the hysteresis: no visible hysteresis was shown for the inflated pressure $0,10,25$, and $40 \mathrm{kPa}$; small hysteresis was shown with the $50 \mathrm{kPa}$. Sensor output with load showed good linearity $\left(r^{2}>0.99\right)$ for the inflated pressure $0,10,25$, and 40. Data for inflated pressure $50 \mathrm{kPa}$ showed lower linearity with average $r^{2}=0.98$. Signals were recorded at $1000 \mathrm{~Hz}$. The system showed good sensitivity with less than $0.05 \mathrm{~N}$ with characterization method found in [24] $(0.0287 \mathrm{~N}$, $0.0263 \mathrm{~N}, 0.0222 \mathrm{~N}, 0.0218 \mathrm{~N}$, and $0.0351 \mathrm{~N}$ for the inflated pressure $0,10,25,40$ and $50 \mathrm{kPa}$ ). Additional filtering can further reduce the signal noise to increase the sensitivity and reliability of the fingertips. Data are shown in Fig. 2 d.

\section{B. Gripper and fingertips control}

We redesigned the GR2 gripper with a gear mechanism instead of a tendon-driven actuation to increase the actuation 
Fig. 5. 3D printed soft cylinders with pneumatic sensors in variation sizes.

accuracy, as well as locked the central triangular link to simplify the grasping stage. Two Dynamixel MX-64T servo motors (ROBOTIS, South Korea) were used to control the gears via a U2D2 USB communication converter with Labview 2018. Fig. 3a. shows the kinematic chain of the gripper during the grasping task. The linkage between P1 and P4 are fixed in this study to make the GR2 gripper perform as a traditional fixed-geometry base two-finger robot gripper. During grasping tasks, the soft air cavities on the fingertips deform when the gripper grasps an object. The sensing data in Fig. 3 shows the demonstrated pressure variations of all four air cavities (L1, $\mathrm{L} 2$ on the left fingertip and R1, R2 on the right fingertip) when grasping a $30 \mathrm{~mm}$ cylindrical object.

The pneumatic diagram in Fig. 4 shows the connection of the four soft air cavities in the fingertips and external hardware. The air cavities were connected with 4 pressure sensors (PSE 543-R06, SMC Corporation, Japan), 5 solenoid valves (Z031C, Sirai, Italy), one air reservoir, and an air pump. A National Instrument DAQ (USB-6341) with Labview 2018 was used to measure the internal air pressure and control the experiment. The internal air pressure of each soft air cavities (L1, L2, R1, R2) was controlled separately with a closed-loop pressure controller. The pressure increase was achieved by a proportional controller with the air pump while a computercontrolled solenoid valve (S5) was used in the system to decrease the pressure. Since the air cavities have a small volume, an air reservoir was used to stabilize the internal pressure without overshoot. When closing the solenoid valves S1, S2, S3, and S4, the internal air mass in the air cavity was maintained. Then, the air cavities L1, L2, R1, and R2 can be used for sensing tasks by measuring the internal pressure variations through the pressure sensors $\mathrm{P} 1, \mathrm{P} 2, \mathrm{P} 3$, and $\mathrm{P} 4$, respectively. When the shape-changing of the fingertips is needed, the controller will be used to inflate the soft air cavities to desired pressure conditions.

\section{Crushing Test with Soft ObJects}

\section{A. Soft testing Objects}

The experiment focuses on demonstrating the sensing capability and repeatability of the tactile sensors during soft object grasping. In order to quantify the impact of the gripper on soft objects, 3D printed soft cylinders with the same concept of pneumatic sensing of the fingertips were used in this study. The soft cylinders are airtight and connected to a pressure sensor where the internal pressure change of the cylinder represents the amount of deformation caused by an
Fig. 6. Type 1 fingertips with only silicone pad and type 2 fingertips with pressurized air cavities

external force. Five soft sensing cylinders with the diameter $D_{0}=10,20,30,40,50 \mathrm{~mm}$, length of $50 \mathrm{~mm}$, and $1.5 \mathrm{~mm}$ thickness were 3D printed using combinations of TangoPlus and VeroClear on an Object $2603 \mathrm{D}$ printer [27] with the material FLX9960-DM (Fig. 5).

\section{B. Gripper position control with tactile sensors}

Two types of fingertips (Fig. 6) were tested to show the comparison results on grasping soft cylindrical objects. Firstly, only position control of the gripper with fingertip type 1 (pure silicone fingertip with no sensor) were used to grasp the soft object to the distance between two fingers $D=0.9 D_{0}$, $D=0.8 D_{0}, D=0.7 D_{0}$, and $D=0.6 D_{0}$, where $D_{0}$ is the initial diameter of the soft object without deformation. The smaller the distance $D$ between the two fingers, the larger deformation will be caused by the grasp. The relative pressure change inside the soft object reflects the deformation quantitatively. During the test, each object was placed in the center of the grippers. This procedure was repeated 5 times for each grasping condition. Secondly, position control with fingertip tactile sensors was enabled with fingertip type 2 to detect the object during grasping. We implemented a closed-loop controller based on the pressure information from the 4 air cavities on the fingertips to control the input angle $\theta$ of the gripper during the test [28]. The controller is described as

$$
\theta_{k}= \begin{cases}\theta_{k-1}+\dot{\theta_{d}} \Delta t & \text { if } P_{k} \leq P_{s} \\ \theta_{k-1} & \text { if } P_{s}<P_{k} \leq P_{t} \\ \theta_{k-1}-\dot{\theta_{d}} \Delta t & \text { if } P_{t}<P_{k}\end{cases}
$$

and

$$
\theta_{\min } \leq \theta_{k} \leq \theta_{\max }
$$

where, $\theta_{k}$ is the present angle of the motor, $P_{k}$ is the highest pressure change among the 4 sensed pressures from L1, L2, $\mathrm{R} 1$, and R2 (to mitigate the grasping position errors), $\dot{\theta}_{d}$ is the reference velocity to open/close gripper, $\Delta t$ is the time step, $P_{s}$ and $P_{t}$ are the lower and higher band pressure (within sensor sensing range, see Fig. 2d), and $\theta_{\min }$ and $\theta_{\max }$ are the minimum and maximum rotation angle of the gripper. During the tactile sensing experiment, $P_{s}$ was set to be $0.1 \mathrm{kPa}$ while $P_{t}$ was set to be $0.12 \mathrm{kPa}$. In the experiment, the signal sampling rate from the pressure sensors was $1000 \mathrm{~Hz}$ and a Median filter with window size of 100 was applied to the signal. We performed 15 trials for each soft object by placing them in the center of the grippers for testing the repeatability of the sensor. 
Fig. 7. The figure shows the relative internal pressure change when feedback control is enabled with tactile sensors on the fingertips during the grasping test. The results with 5 soft cylinders $1-5$ with the diameter $D_{0}=10,20,30,40,50 \mathrm{~mm}$ are shown in sub-figures a-e, respectively, a standard deviation of 15 trials is shown. The y-axis label is same for sub-figures a-e.

Fig. 8. The relative internal pressure change of the soft objects during stable grasping. The result of tactile sensors on fingertips are shown with comparison to results of set gripper positions $D=0.9 D_{0}, 0.8 D_{0}, 0.7 D_{0}, 0.6 D_{0}$. The result with 5 soft cylinders $1-5$ with the diameter $D_{0}=10,20,30,40,50 \mathrm{~mm}$ are shown in sub-figures a-e, respectively,a standard deviation of 15 trials is shown.The y-axis label is same for sub-figures a-e.

For only the position control experiment, the input angle $\theta$ of the gripper was calculated with given distance D using the bilateration method [29]. Two vectors $\mathbf{p}_{4,5}$ and $\mathbf{p}_{4,3}$ are connecting point $P_{4}$ to $P_{5}$ and point $P_{4}$ to $P_{3}$, as shown in Fig. 3 . These two vectors can form a bilateration matrix, $\mathbf{Z}_{4,5,3}$, and then $\mathbf{p}_{4,3}$ can be computed as:

$$
\mathbf{p}_{4,3}=\mathbf{Z}_{4,5,3} \mathbf{p}_{4,5}
$$

where

$$
\mathbf{Z}_{4,5,3}=\frac{1}{2 s_{4,5}}\left[\begin{array}{cc}
s_{4,3}+s_{4,5}-s_{5,3} & -4 \Delta_{4,5,3} \\
4 \Delta_{4,5,3} & s_{4,3}+s_{4,5}-s_{5,3}
\end{array}\right],
$$

and

$$
\Delta_{4,5,3}= \pm \frac{1}{4} \sqrt{\left(s_{4,3}+s_{4,5}+s_{5,3}\right)^{2}-2\left(s_{4,3}^{2}+s_{4,5}^{2}+s_{5,3}^{2}\right)},
$$

with $\Delta_{4,5,3}$ being the oriented area of the triangle defined by points $P_{4}, P_{5}$, and $P_{3}\left(\triangle_{453}\right)$. The positive or negative of it implies that $\mathbf{p}_{4,3}$ can point in one of two directions. When $P_{3}$ is to the right of vector $\mathbf{p}_{4,5}$, the oriented area is negative; otherwise it is positive. With the same equation, $P_{2}$ can be computed with the same theory by points $P_{5}, P_{3}$, and $P_{2}\left(\triangle_{532}\right)$ :

$$
P_{2}=P_{5}+\mathbf{Z}_{5,3,2} \mathbf{p}_{5,3}
$$

A detailed description of these formulae can be found in [29], [30].

\section{Results}

Fig. 7 shows the crushing test results of the fingertip with tactile sensors. During grasping, the internal pressure of the grasped soft objects exhibit a gradual increase until the increased contact pressure triggered the controller to stabilize. For all the trials, the sensor shows good consistency and repeatability with a average standard deviation error of $0.24,0.34,0.42,0.25$, and $0.08 \mathrm{kPa}$ for soft cylinders $D_{0}=10,20,30,40,50 \mathrm{~mm}$, respectively. When the fingertips interact with a soft object during grasping tasks, the relative sensitivity of the tactile sensors is affected by the stiffness of the fingertips and the stiffness of the soft object. When the stiffness of the fingertips is fixed, as shown in the experiments, the tactile sensors are more sensitive when the object is stiffer. In the study, although all of the soft cylinders are 3D printed with the same material and same thickness, the smaller diameter cylinders would have higher strain energy density due to the structure. Thus, smaller cylinders exhibit higher stiffness. On the other hand, smaller volume soft cylinders would cause a larger pressure change with the same amount of deformation ( $P V=n R T$ where $n, R, T$ are constants). Considering the two factors (structure stiffness and volume), soft cylinder $2 D_{0}=20 \mathrm{~mm}$ exhibits the highest pressure variation during grasping in the experiment.

The stabilized pressure data from the soft objects with closing gripper distance $D=0.9 D_{0}, 0.8 D_{0}, 0.7 D_{0}, 0.6 D_{0}$ are compared with the test when tactile sensors are used for control with pressure limit sets between $P_{s}=0.1 \mathrm{kPa}$ and 
Fig. 9. (a) Experiment setup for the escaping test. The robot arm only moves up and down with gradually increased speed and acceleration after the object is secured in hand. (b) Two $0.5 \mathrm{~N}$ standard calibration weights were attached on the soft object.

TABLE I

SET PRESSURES FOR THE ESCAPING TEST

\begin{tabular}{|l|l|l|l|l|l|}
\hline Soft object diameter $D_{0}[\mathrm{~mm}]$ & 10 & 20 & 30 & 40 & 50 \\
\hline Set pressure $[\mathrm{kPa}]$ & 2 & 1.2 & 1.5 & 1 & 0.5 \\
\hline
\end{tabular}

$P_{t}=0.12 \mathrm{kPa}$. The relative internal pressure comparison results between fingertips with and without tactile sensors are shown in Fig. 8. It proves that deformation can be kept below 0.7 of the original diameter for all 5 different sized soft cylinders under current set limit conditions. The set limit condition $P_{S}$ and $P_{t}$ can be tuned for lower or higher force grasp. The sensitivity of the fingertips can also be increased by reducing the material stiffness.

\section{Escaping Test with High-SPEed Manipulation}

\section{A. Experiment setup}

In the industrial environment, products are commonly picked and placed from one location to another location. Grasping the product with a gripper and ensuring the product "follows" the trajectory of the manipulator is essential. With only force closure by contact friction, the grasping normally requires relatively high force, which makes it inappropriate for soft and fragile products. By having form closure or caging grasps, the capability of the object to "follow" the manipulator without escaping can be significantly increased. In this experiment, we attach the gripper on a UR5 robot arm (Universal Robots, Denmark) to test the performance of the fingertips during high-speed manipulations. We expect the type 2 fingertips with active deformation can secure the grasping object better under high-speed manipulation compare to type 1 fingertips with only silicone pads.

Fig. 9 shows the setup of the experiment. The robot arm was programmed to do a sinusoidal movement with gradually increased speed and acceleration after the gripper grasped the object and secured it in hand. Type 2 fingertips with active shaping changing ability and type 1 fingertips with only silicone pads were tested separately with soft objects with the diameter $D_{0}=10,20,30,40,50 \mathrm{~mm} .0 .5 \mathrm{~N}$ standard calibration
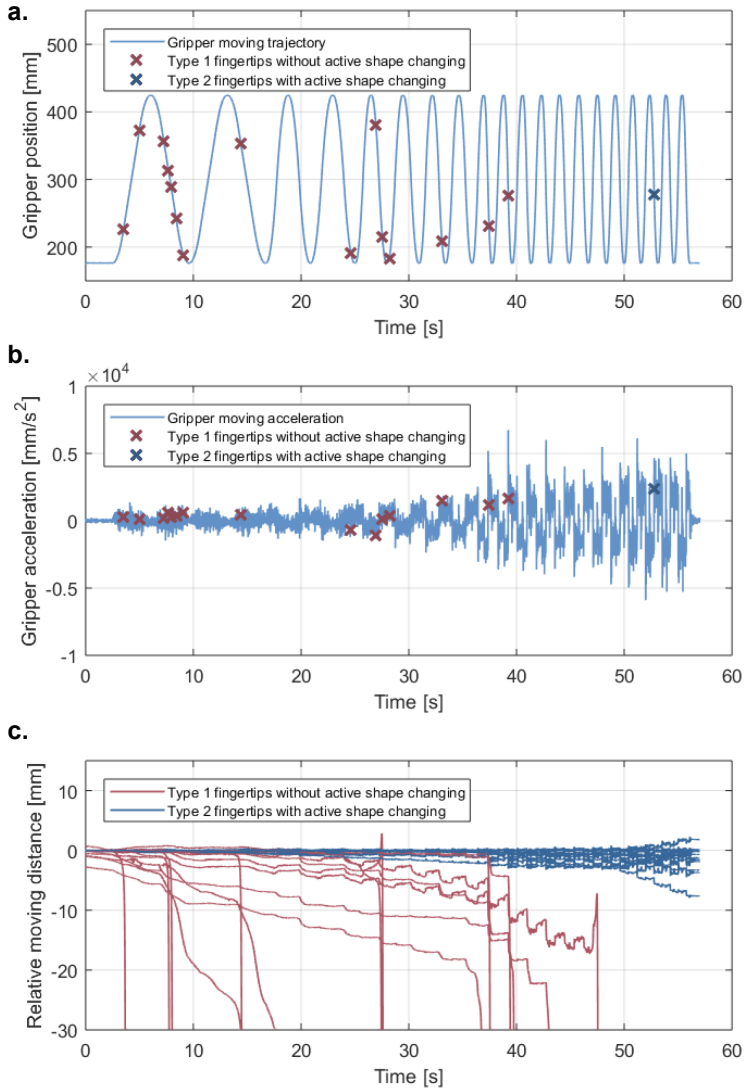

Fig. 10. (a) Gripper position during the escaping test. (b) Gripper acceleration during the escaping test. (c) Relative moving distance between the gripper and object during the escaping test. Red and blue cross marks show the position and acceleration where the object loss in contact with type 1 fingertips and type 2 fingertips, respectively. 15 trials for each fingertip are shown.

weights were attached on each side of the soft object to add extra initial inertia (shown in Fig. 9 b). Relative moving distance calculated based on the distance change between the gripper and object were used to evaluate the grasping robustness. Motion tracking cameras (Flex 3 OptiTrack, NaturalPoint, Inc., USA) were used to record the gripper and object position during manipulations with pre-attached reference markers. The internal pressure change of the soft object was recorded and used as a reference to ensure equal force was applied to the object between type 1 and type 2 fingertips. Before each manipulation, type 2 fingertip cavities were inflated to a certain size to adapt to the object shape until the soft object reached the set pressure. Active shape-changing of the fingertips is needed to ensure the form-closure created for objects with different diameters. Table I shows the set pressures of the 5 soft objects in the test. Each soft object was tested 3 times for repetition.

\section{B. Result}

Fig. 10 a. shows the gripper position represented by the end-effector of the UR5 robot arm as they move together as a rigid body. Fig. 10 b. shows the acceleration of the gripper during the test. Fig. $10 \mathrm{c}$. shows the relative moving distance between the object and the gripper with 15 trials of test for 
Fig. 11. (a) Form-closure model of the inflated fingertips with 4 Kelvin-Voight models. (b) Example sensing data from the 4 pressurized soft cavities L1, $\mathrm{L} 2, \mathrm{R} 1$, and R2 for the time period of $40 \mathrm{~s}$ to $55 \mathrm{~s}$ of one trial of soft object $D_{0}=30 \mathrm{~mm}$ test. Moving trajectory and acceleration during the period are shown in Fig. 10.

pure silicone fingertips shown in red and dual-soft fingertips shown in blue for the 5 different soft objects. When the relative distance between the object and gripper is greater than $5 \mathrm{~mm}$, the object will be considered as "escape" during the test. All the tests carried out with fingertips type 2 , with active changed shape, successfully secured the object during the high-speed manipulation test with accelerations more than $5 \mathrm{~m} / \mathrm{s}^{2}$. Only 1 out of the 15 trials experiment for fingertip type 2 had displacement larger than $5 \mathrm{~mm}$. In contrast, none of the 15 trials with fingertips type 1 passed the test, while the object started to show "offset" when the gripper starts moving and "loss contact" at manipulation accelerations as low as $0.1 \mathrm{~m} / \mathrm{s}^{2}$. The positions where the "escape" happened are marked with cross marks in Fig. 10 a. and b. A clear oscillation can be observed from the relative moving distance data, as shown in Fig. $10 \mathrm{c}$.

During the manipulation tasks with fingertips type 1, friction is mainly used to secure the object in hand $F_{f}-m g=m a_{t}$ and $F_{f} \leqslant \mu_{s} N$, where $F_{f}$ is the friction between fingertips and the object, $m$ is the mass of the object, $a_{t}$ is the object acceleration in vertical direction, $\mu_{s}$ is the static coefficient of friction, and $N$ is the applied normal force from the grasping. The object starts losing contact with the gripper when its inertia overcomes the static friction when the acceleration is high. The object will be secured again when the gripper reduces its speed when approaching the peak points. The oscillations observed in Fig. 10 c. for type 1 fingertips manipulations reflects these short-period of secured grasps.

For the manipulation tasks with fingertips type 2, small oscillations can also be observed, particularly during high acceleration periods. However, the oscillations are relatively smaller in amplitudes compare to fingertips type 1 , and the causes for the oscillations of the two types of fingertips are different. The form-closure structure formed by the active shape-changing fingertips will also act as soft dampers to reduce the contact force between the gripper and soft object. Viscoelastic material such as silicone can be modelled by connecting a spring and a dashpot in parallel as Kelvin-Voight model [31]. Fig. 11a. shows the model of grasping with form- closure fingertips. During high accelerations, the fingertips will thus deform according to the direction and amplitude of the acceleration. The form-closure structure will then behave like a tight caging with shock absorbers which allow the object to move during dynamic conditions. Extra protection of the soft object can be provided with smaller contact force compared to rigid form-closure grippers. This explains the oscillation of the relative distance between the object and gripper for type 2 fingertips during high-acceleration manipulations.

In addition, the inflated fingertips can also sense the force between the object and different area of the fingertips during the manipulation. Fig. 11 b. shows the sensor reading from L1, L2, R1, and R2 pressurized air cavities during a period of manipulation. The data is shown by one trial of soft object 3 and $D_{0}=30 \mathrm{~mm}$ manipulation test for the period of 40 to $55 \mathrm{~s}$. The sensor reading data reflects the oscillations in the relative moving distance, as shown in Fig. $10 \mathrm{c}$. Clear oscillations of the relative pressure can be observed when higher and lower forces were applied to the object. In the experiment, the cylindrical object was placed in the center of the gripper. Due to the in-hand location of the cylindrical object and the sinusoidal movement, the top two pressurized air cavities L1 and R1 show similar trend while the bottom two pressurized air cavities L2 and R2 show opposite trends. When the object moves upward relative to the gripper, the top two sensor readings increase while the bottom two sensor readings decrease. The curved morphology of the fingertips allows the tactile sensors to sense forces in different configuration compared to its original non-inflated states. The in-hand position and force distribution of the object can, therefore, be monitored during the manipulation.

\section{Conclusions}

This paper presents the concept of a soft fingertip design with the dual function of tactile sensing and active shapechanging. The fingertips are integrated on a traditional grasper with four-bar-linkage fingers based on the GR2 robot gripper, with pressure feedback control to enable safe grasping with soft and delicate objects. The proposed fingertips achieve 
form closure by inflating different sections to change its shape during grasping. With the soft form-closure structure, the gripper can not only secure the object for high-speed manipulation but also reduce the increased contact impact force caused by inertia. In addition, when the fingertip is inflated, the change of its morphology would change the way the fingertip senses, from flat to a curved baseline.

In this study, we limited the objects to soft sensorised cylindrical objects with different diameters to clearly demonstrate the dual function of active shape-changing and sensing of the fingertip. Objects from the YCB grasping test were also grasped with a success rate of $100 \%$ (repeated 3 times) to show the grasping reliability and repeatability. Selected testing videos are also included in the supplementary materials[32]. One limitation of the current design is that actuation of the fingertip for shape-changing of a single air cavity cannot be achieved simultaneously with its sensing function. Further detailed calibrations of the fingertips and design of more specific controllers are needed for future applications.

Rigid grippers have the advantages of low cost, simple but precise control, and high reliability while soft robotic grippers are able to safely interact with delicate objects due to its high adaptability and low contact pressure. The fingertip design we proposed combined the advantages of rigid and soft robotic grippers by: 1) using rigid mechanism for precise general grasping and manipulation, 2) applying soft gripper actuation in a local contact level to retain safe interaction with the object, and 3) integrating soft tactile sensing on the fingertip for feedback control. Grippers with such dualfunction fingertips have the advantage to be used in food industries with food products that are easy to bruise, tear, or deform. Compared to the prehensile approach by most soft robotic grippers, the form closure (which can behave like a tight caging with shock absorbers) achieved by the fingertips active shape-changing provides the potential to manipulate the object without immobilizing it. Future study will focus on using the shape-changing and tactile sensing ability of the fingertip for dexterous in-hand manipulation.

\section{REFERENCES}

[1] P. Y. Chua, T. Ilschner, and D. G. Caldwell, "Robotic manipulation of food products - a review," Industrial Robot, vol. 30, pp. 345-354, 2003.

[2] F. Bader and S. Rahimifard, "Challenges for industrial robot applications in food manufacturing," ACM International Conference Proceeding Series, 2018.

[3] S. Birrell, J. Hughes, J. Y. Cai, and F. Iida, "A field-tested robotic harvesting system for iceberg lettuce," Journal of Field Robotics, vol. 0, no. 0, 2019.

[4] J. Gray and S. Davis, "Robotics in the food industry: an introduction," in Robotics and Automation in the Food Industry, ser. Woodhead Publishing Series in Food Science, Technology and Nutrition, D. G. Caldwell, Ed. Woodhead Publishing, 2013, pp. 21 - 35.

[5] C. Blanes, M. Mellado, C. Ortiz, and A. Valera, "Review. technologies for robot grippers in pick and place operations for fresh fruits and vegetables," Spanish Journal of Agricultural Research, vol. 9, no. 4, pp. 1130-1141, 2011

[6] C. Blanes, M. Mellado, and P. Beltran, "Novel additive manufacturing pneumatic actuators and mechanisms for food handling grippers," Actuators, vol. 3, no. 3, pp. 205-225, 2014.

[7] P. Pedro, C. Ananda, P. B. Rafael, A. R. Carlos, and B. C. Alexandre, "Closed structure soft robotic gripper," 2018 IEEE International Conference on Soft Robotics, RoboSoft 2018, vol. 2, pp. 66-70, 2018.
[8] R. Sam and S. Nefti, "A new design approach of robotic gripper for reducing operating cost for handling food product," 2010 IEEE 9th International Conference on Cybernetic Intelligent Systems, CIS 2010, pp. 1-5, 2010.

[9] K. K. Chan and N. C. Cheung, "A novel two-finger variable-reluctance gripper for high-speed grasping of delicate objects: An implementation case study," IEEE Transactions on Industrial Electronics, vol. 52, no. 6 , pp. 1705-1707, 2005.

[10] B. Ward-Cherrier, N. Rojas, and N. F. Lepora, "Model-Free Precise inHand Manipulation with a 3D-Printed Tactile Gripper," IEEE Robotics and Automation Letters, vol. 2, no. 4, pp. 2056-2063, 2017.

[11] F. Ilievski, A. D. Mazzeo, R. F. Shepherd, X. Chen, and G. M. Whitesides, "Soft robotics for chemists," Angewandte Chemie - International Edition, vol. 50, no. 8, pp. 1890-1895, 2011.

[12] E. Brown, N. Rodenberg, J. Amend, A. Mozeika, E. Steltz, M. R. Zakin, H. Lipson, and H. M. Jaeger, "Universal Robotic Gripper based on the Jamming of Granular Material," 2010.

[13] Q. Lu and N. Rojas, "On soft fingertips for in-hand manipulation: Modeling and implications for robot hand design," IEEE Robotics and Automation Letters, vol. 4, no. 3, pp. 2471-2478, 2019.

[14] Z. Wang, D. S. Chathuranga, and S. Hirai, "3D printed soft gripper for automatic lunch box packing," 2016 IEEE International Conference on Robotics and Biomimetics, ROBIO 2016, pp. 503-508, 2016.

[15] A. Petterson, T. Ohlsson, D. G. Caldwell, S. Davis, J. O. Gray, and T. J. Dodd, "A Bernoulli principle gripper for handling of planar and 3D (food) products," Industrial Robot, vol. 37, no. 6, pp. 518-526, 2010.

[16] V.-D. Nguyen, "Constructing force- closure grasps," The International Journal of Robotics Research, vol. 7, no. 3, pp. 3-16, 1988.

[17] A. Bicchi and C. E. Piaggio, "On the Closure Properties of Robotic Grasping," J. of Robotics Research, 1993.

[18] M. Honarpardaz, M. Tarkian, J. Ölvander, and X. Feng, "Finger design automation for industrial robot grippers: A review," Robotics and Autonomous Systems, vol. 87, pp. 104-119, 2017.

[19] H. Song, M. Y. Wang, and K. Hang, "Fingertip Surface Optimization for Robust Grasping on Contact Primitives," IEEE Robotics and Automation Letters, vol. 3, no. 2, pp. 742-749, 2018.

[20] N. Herzig, P. Maiolino, F. Iida, and T. Nanayakkara, "A Variable Stiffness Robotic Probe for Soft Tissue Palpation," IEEE Robotics and Automation Letters, vol. 3, no. 2, pp. 1168-1175, 2018.

[21] C. Jackson, H. Vermaak, and G. Jordaan, "Force sensing for dynamic gripping, using a piezoelectric sensor," IEEE International Symposium on Industrial Electronics, pp. 1371-1376, 2007.

[22] A. Petrovskaya, O. Khatib, S. Thrun, and A. Y. Ng, "Bayesian estimation for autonomous object manipulation based on tactile sensors," Proceedings - IEEE International Conference on Robotics and Automation, vol. 2006, pp. 707-714, 2006.

[23] H. Fischer and R. Wolf, "Tactile pressure sensor," Nov. 16 1999, uS Patent 5,983,725.

[24] Y. Tenzer, L. P. Jentoft, and R. D. Howe, "The feel of mems barometers: Inexpensive and easily customized tactile array sensors," IEEE Robotics \& Automation Magazine, vol. 21, no. 3, pp. 89-95, 2014.

[25] N. Rojas, R. R. Ma, and A. M. Dollar, "The GR2 Gripper: An Underactuated Hand for Open-Loop In-Hand Planar Manipulation," IEEE Transactions on Robotics, vol. 32, no. 3, pp. 763-770, 2016.

[26] J. Kim, A. Alspach, and K. Yamane, "3d printed soft skin for safe human-robot interaction," in 2015 IEEE/RSJ International Conference on Intelligent Robots and Systems. IEEE, 2015, pp. 2419-2425.

[27] Stratasys, "Digital Materials ( DMs ) Data Sheet," 2013. [Online]. Available: http://stratasys.com/materials/polyjet/digital-materials

[28] T. Nanayakkara, A. Jiang, M. del Rocío Armas Fernández, H. Liu, K. Althoefer, and J. Bimbo, "Stable grip control on soft objects with time-varying stiffness," IEEE Transactions on Robotics, vol. 32, no. 3, pp. 626-637, June 2016.

[29] N. Rojas, "Distance-based formulations for the position analysis of kinematic chains," Ph.D. dissertation, Universitat Politècnica de Catalunya, 2012.

[30] N. Baron, A. Philippides, and N. Rojas, "A Novel Kinematically Redundant Planar Parallel Robot Manipulator With Full Rotatability," Journal of Mechanisms and Robotics, vol. 11, no. 1, 112018.

[31] I. T. Jaswal and A. Mahmood, "Robotic gripping using soft finger tips," ICIEECT 2017 - International Conference on Innovations in Electrical Engineering and Computational Technologies 2017, Proceedings, pp. 1-5, 2017

[32] B. Calli, A. Walsman, A. Singh, S. Srinivasa, P. Abbeel, and A. M. Dollar, "Benchmarking in manipulation research: Using the yale-cmuberkeley object and model set," IEEE Robotics \& Automation Magazine, vol. 22 , no. 3 , p. 36-52, Sep 2015. 